QUARTERLY OF APPLIED MATHEMATICS

VOLUME LXVI, NUMBER 3

SEPTEMBER 2008, PAGES 499-520

S 0033-569X(08)01088-2

Article electronically published on July 2, 2008

\title{
SOLVING SPDES DRIVEN BY COLORED NOISE: A CHAOS APPROACH
}

\author{
BY \\ S. V. LOTOTSKY (Department of Mathematics, USC, Los Angeles, California 90089) \\ AND \\ K. STEMMANN (Department of Mathematics, USC, Los Angeles, California 90089)
}

\begin{abstract}
An Itô-Skorokhod bilinear equation driven by infinitely many independent colored noises is considered in a normal triple of Hilbert spaces. The special feature of the equation is the appearance of the Wick product in the definition of the Itô-Skorokhod integral, requiring innovative approaches to computing the solution. A chaos expansion of the solution is derived and several truncations of this expansion are studied. A recursive approximation of the solution is suggested and the corresponding approximation error bound is computed.
\end{abstract}

1. Introduction. Stochastic differential equations driven by Gaussian white noise are well-studied; see, for example, the book [35] for ordinary differential equations and the book [37] for equations with partial derivatives. The underlying stochastic process in these equations is the standard Brownian motion $W$, which is a square-integrable Gaussian martingale with continuous trajectories and independent increments. A lot less is known about equations driven by colored noise, when the underlying process is still Gaussian, but no longer has independent increments. An important example is the fractional Brownian motion $W^{H}, H \in(0,1)$, which coincides with the standard Brownian motion $W$ for $H=1 / 2$ and is not a semi-martingale for all $H \neq 1 / 2$. It is the lack of the semi-martingale property that makes the analysis difficult at the very basic level, the definition of the corresponding stochastic integral. Several versions of the stochastic integral with respect to $W^{H}$ have been proposed 1, 12, 13, 14, 23, 26, 38. Unlike the standard Brownian motion, different approaches such as Itô-type vs. Stratonovichtype integral or path-wise vs. mean-square definition, become much more difficult to reconcile. The paper by V. Pipiras and M. Taqqu [36] describes the main difference

Received May 15, 2007.

2000 Mathematics Subject Classification. Primary 60H15; Secondary 35R60, 60H40.

Key words and phrases. Generalized random fields, Malliavin calculus, Skorokhod integral, Wiener chaos.

The first author acknowledges support from the NSF CAREER award DMS-0237724.

The work of K. Stemmann was partially supported by the NSF Grant DMS-0237724.

E-mail address: lototsky@math.usc.edu 
between integration with respect to $W$ and $W^{H}$ for $H \neq 1 / 2$ and demonstrates the resulting technical difficulty. Beside a purely theoretical interest, fractional Brownian motion appears to be a natural replacement of the standard Brownian motion in certain applied problems [3, 4, 5, 9, 19, 22. In many such problems, it is possible to avoid most of the technical issues related to the definition of the stochastic integral by considering additive noise.

One way to streamline the analysis of differential equations driven by multiplicative noise (also known as bilinear equations) is to use the Wick product. It is known [18] that, with standard Brownian motion, the Wick product and the usual calculus lead to the same results as the usual product and the Itô calculus. While the use of the Wick product has been questioned as a modeling tool for certain applications in economics and finance [8], it is still an effective tool for theoretical investigations, corresponding to the Itô-Skorokhod integral in the white noise analysis.

A successful study of differential equations driven by multiplicative colored noise also requires a convenient representation of the underlying Gaussian process. Traditionally, a Gaussian process is defined by its mean and covariance functions, but then the definition of the integral immediately leads to a number of technical conditions on these functions 22. An alternative definition is possible 29] by combining the ideas from the theory of generalized Gaussian fields [15, 30, the white noise theory [17, 18, and the Malliavin calculus 31, 34. This approach to stochastic integration is used in this paper and is outlined in Section 2

Numerical methods for stochastic ordinary differential equations driven by white noise are a well-developed subject [24, 32. Equations with partial derivatives have been mostly studied in connection with optimal nonlinear filtering [6, 7, 16, 20, 21]. In the end, all these numerical methods have obvious counterparts in the numerical analysis of the deterministic equations (Galerkin method, Euler method, finite difference and finite element methods, operator splitting method, etc.).

The main difficulty in the numerical analysis of equations driven by colored noise is the use of the Wick product: unlike the usual product, the Wick product is not an operation readily performed by a computer. Since the Wick product is relatively easy to compute for Hermite polynomials of Gaussian random variables, an implementation of this operation should be based on the chaos expansion, and then a truncation of this expansion becomes a natural numerical approximation of the solution.

While a truncated chaos expansion has been investigated before as an approximation of the solution of a stochastic equation, in particular, for equations of optimal nonlinear filtering [10, 27, etc.], this approximation was always just another possibility of solving the equation numerically. By contrast, for equations driven by colored noise, chaos expansion appears to be the only possibility to compute the solution. The chaos expansion of the solution of a bilinear equation driven by infinitely many independent colored noises is studied below in Section 3 various truncations of this expansion are studied in Section 4.

2. Colored noise. Let $(\Omega, \mathcal{F}, \mathbb{P})$ be a complete probability space. 
Definition 2.1. (a) A colored noise $\mathfrak{X}$ on $L_{2}((0, T))$ with the covariance operator $\mathcal{R}$ is a collection of zero-mean Gaussian random variables $\mathfrak{X}(f), f \in L_{2}((0, T))$, with the property

$$
\mathbb{E}(\mathfrak{X}(f) \mathfrak{X}(g))=\int_{0}^{T}(\mathcal{R} f)(s) g(s) d s, f, g \in L_{2}((0, T)),
$$

where $\mathcal{R}$ is a bounded linear operator on $L_{2}((0, T))$.

(b) A representation operator of $\mathfrak{X}$ is a bounded linear operator $\mathcal{K}$ on $L_{2}((0, T))$ such that $\mathcal{K K}^{*}=\mathcal{R}$, where $\mathcal{K}^{*}$ is the adjoint of $\mathcal{K}$.

REMARK 2.2. (a) The white noise on $L_{2}((0, T))$ corresponds to $\mathcal{R}=I$, the identity operator [17].

(b) Since $\mathcal{R}$ is a selfadjoint nonnegative operator, a representation operator always exists.

For $t>0$ let

$$
\chi_{t}(s)= \begin{cases}1, & 0 \leq s \leq t \\ 0, & \text { otherwise }\end{cases}
$$

denote the characteristic function of the interval $[0, t]$.

EXAMPLE 2.3. If $\mathfrak{X}$ is white noise, then direct computations show that $W(t)=\mathfrak{X}\left(\chi_{t}\right)$ is a standard Brownian motion and

$$
\mathfrak{X}(f)=\int_{0}^{T} f(s) d W(s), f \in L_{2}((0, T)) .
$$

Example 2.4 (Ornstein-Uhlenbeck Noise). For $b>0$ and $t, s \in[0, T]$ define

$$
K(t, s)=-b e^{-b(t-s)} .
$$

Then the operator

$$
\mathcal{K}: f(t) \mapsto(\mathcal{K} f)(t)=f(t)+\int_{0}^{t} K(t, s) f(s) d s
$$

is bounded on $L_{2}((0, T))$ :

$$
\int_{0}^{T}|(\mathcal{K} f)(s)|^{2} d s \leq(1+\sqrt{b T})^{2} \int_{0}^{T}|f(s)|^{2} d s .
$$

If $\mathfrak{X}$ is the colored noise corresponding to the representation operator $\mathcal{K}$, then a straightforward computation shows that $\mathfrak{X}\left(\chi_{t}\right), t \in(0, T)$, is the Ornstein-Uhlenbeck process with covariance

$$
\mathbb{E}\left(\mathfrak{X}\left(\chi_{t}\right) \mathfrak{X}\left(\chi_{s}\right)\right)=e^{-b|t-s|} .
$$

Accordingly, we call $\mathfrak{X}$ the Ornstein-Uhlenbeck noise with parameter $b$.

ExAmple 2.5 (Fractional White Noise). For $H \in(1 / 2,1)$ and $t, s \in[0, T]$ define

$$
K(t, s)=C_{H}\left(H-\frac{1}{2}\right)\left(\frac{t}{s}\right)^{\frac{1}{2}-H}(t-s)^{H-\frac{3}{2}} \chi_{t}(s),
$$

where

$$
C_{H}=\left(\frac{2 H \Gamma\left(\frac{3}{2}-H\right)}{\Gamma\left(H+\frac{1}{2}\right) \Gamma(2-2 H)}\right)^{\frac{1}{2}}
$$


and $\Gamma$ is the Gamma function. Then the operator

$$
\mathcal{K}: f(t) \mapsto(\mathcal{K} f)(t)=\int_{0}^{t} K(t, s) f(s) d s
$$

is bounded on $L_{2}((0, T))[29,33$ :

$$
\int_{0}^{T}|(\mathcal{K} f)(s)|^{2} d s \leq \frac{H(2 H-1) \Gamma\left(H-\frac{1}{2}\right)}{\Gamma\left(H+\frac{1}{2}\right)} T^{2 H-1} \int_{0}^{T}|f(s)|^{2} d s .
$$

If $\mathfrak{X}$ is the colored noise corresponding to the representation operator $\mathcal{K}$, then $\mathfrak{X}\left(\chi_{t}\right), t \in$ $(0, T)$, is the fractional Brownian motion with Hurst parameter $H$ 33. Accordingly, we call $\mathfrak{X}$ the $H$-fractional white noise.

In general, if $\mathfrak{X}$ is a colored noise on $L_{2}((0, T))$, then $X(t)=\mathfrak{X}\left(\chi_{t}\right)$ is a zero-mean Gaussian process. Thus, one can interpret $\mathfrak{X}$ as a collection of integrals $\int_{0}^{T} f(s) d X(s)$ for deterministic $f \in L_{2}((0, T))$. Our study of bilinear equations (linear equations with multiplicative noise) requires an extension of $\mathfrak{X}$ to random $f$ and is based on the following generalization of (2.2).

Proposition 2.6. For every colored noise $\mathfrak{X}$ on $L_{2}((0, T))$ with a representation operator $\mathcal{K}$, there exists a unique standard Brownian motion $W=W(t)$ such that

$$
\mathfrak{X}(f)=\int_{0}^{T}\left(\mathcal{K}^{*} f\right)(s) d W(s), f \in L_{2}((0, T)) .
$$

Proof. Relation (2.4) certainly defines a colored noise on $L_{2}((0, T))$; we omit the technical proof that the corresponding Brownian motion can be found for every colored noise $\mathfrak{X}[29$.

Definition 2.7. A pair $(\mathcal{K}, W)$, where $\mathcal{K}$ is a bounded linear operator on $L_{2}((0, T))$ and $W$ is a standard Brownian motion, is called a representation of the colored noise $\mathfrak{X}$ if (2.4) holds.

For random $f$, we now define $\mathfrak{X}(f)$ according to (2.4), where the stochastic integral is understood in the Itô-Skorokhod sense [34]. An equivalent, but less convenient, definition of $\mathfrak{X}(f)$ for random $f$ is possible in intrinsic terms without using a representation of $\mathfrak{X}[29]$.

3. Chaos solution: Existence and regularity. Let $(\Omega, \mathcal{F}, \mathbb{P})$ be a complete probability space and $\mathfrak{X}_{\ell}, \ell \geq 1$, a collection of independent colored noises on $L_{2}((0, T))$.

In this section we study the equation

$$
u(t)=u_{0}+\int_{0}^{t}(\mathcal{A} u(s)+F(s)) d s+\sum_{\ell=1}^{\infty} \mathfrak{X}_{\ell}\left(\chi_{t} \mathcal{B}_{\ell} u+\chi_{t} G_{\ell}\right),
$$

$t \in[0, T]$, in a normal triple $\left(\mathbf{X}, \mathbf{H}, \mathbf{X}^{\prime}\right)$ of Hilbert spaces. In particular, we assume that $\mathcal{A}$ and each $\mathcal{B}_{\ell}$ are bounded linear operators from $\mathbf{X}$ to $\mathbf{X}^{\prime}, u_{0} \in L_{2}\left(\Omega ; \mathbf{X}^{\prime}\right), F, G_{\ell} \in$ $L_{2}\left(\Omega ; L_{2}\left((0, T) ; \mathbf{X}^{\prime}\right)\right)$.

REMARK 3.1. While it is tempting to rewrite (3.1) as

$$
u(t)=u_{0}+\int_{0}^{t}(\mathcal{A} u(s)+F(s)) d s+\sum_{\ell=1}^{\infty} \int_{0}^{t}\left(\mathcal{B}_{\ell} u(s)+G_{\ell}(s)\right) d X_{\ell}(s),
$$


where $X_{\ell}(t)=\mathfrak{X}_{\ell}\left(\chi_{t}\right)$, we will use a more rigorous form (3.1).

By analogy with equations driven by white noise [37, we define a variational solution of (3.1) as a random element $u$ with values in $L_{2}\left(\Omega ; L_{2}((0, T) ; \mathbf{X})\right)$ such that, for every $v \in \mathbf{X}$, the equality

$$
(u(t), v)_{\mathbf{H}}=\left\langle u_{0}, v\right\rangle+\int_{0}^{t}\langle(\mathcal{A} u(s)+F(s)), v\rangle d s+\sum_{\ell=1}^{\infty} \mathfrak{X}_{\ell}\left(\chi_{t}\left\langle\mathcal{B}_{\ell} u+G_{\ell}, v\right\rangle\right)
$$

holds in $\mathbf{X}^{\prime}$ on the same set of probability one for all $t \in[0, T]$; by $\langle\cdot, \cdot\rangle$ we denote the duality between $\mathbf{X}^{\prime}$ and $\mathbf{X}$ relative to the inner product $(\cdot, \cdot)_{\mathbf{H}}$ of $\mathbf{H}$.

Unfortunately, the current development of the colored noise calculus is not sufficient to establish existence of a variational solution of (3.1). Accordingly, we introduce a weaker notion of solution, called a chaos solution, using a Fourier series expansion in the space of square integrable random processes.

We start with some auxiliary constructions. Let $\left(\mathcal{K}_{\ell}, W_{\ell}\right)$ be a representation of the colored noise $\mathfrak{X}_{\ell}$ and let $\left\{h_{k}, k \geq 1\right\}$ be an orthonormal basis in $L_{2}((0, T))$. Define the random variables

$$
\xi_{k \ell}=\int_{0}^{T} h_{k}(t) d W_{\ell}(t) .
$$

Let $\mathcal{J}$ be the collection of multi-indices $\alpha=\left\{\alpha_{k \ell}, k, \ell \geq 1\right\}$. Each $\alpha \in \mathcal{J}$ has nonnegative integer elements $\alpha_{k \ell}$ and $\sum_{k, \ell} \alpha_{k \ell}<\infty$. We will use the notation

$$
|\alpha|=\sum_{k, \ell} \alpha_{k \ell}, \alpha !=\prod_{k, \ell} \alpha_{k \ell} !
$$

By (0) we denote the multi-index $\alpha$ with $|\alpha|=0$ and by $\epsilon_{i j}$, the multi-index $\alpha$ with $|\alpha|=1$ and $\alpha_{i j}=1$.

REMARK 3.2. If there is only one colored noise, then the entries of $\alpha$ have only one index: $\alpha=\left\{\alpha_{k}, k \geq 1\right\}$.

For $\alpha \in \mathcal{J}$ define

$$
\xi_{\alpha}=\prod_{k, \ell \geq 1} \frac{H_{\alpha_{k \ell}}\left(\xi_{k \ell}\right)}{\sqrt{\alpha_{k \ell} !}}
$$

where, for an integer $n \geq 0, H_{n}=H_{n}(t)$ is the $n$-th Hermite polynomial

$$
H_{n}(t)=(-1)^{n} e^{t^{2} / 2} \frac{d^{n}}{d t^{n}} e^{-t^{2} / 2}
$$

Recall the definition of the Wick product [18]:

$$
H_{m}\left(\xi_{i j}\right) \diamond H_{n}\left(\xi_{k \ell}\right)= \begin{cases}H_{m+n}\left(\xi_{i j}\right), & \text { if } i=k \text { and } j=\ell, \\ H_{m}\left(\xi_{i j}\right) H_{n}\left(\xi_{k \ell}\right), & \text { otherwise. }\end{cases}
$$

In particular, for $m \geq 1$,

$$
H_{m}\left(\xi_{k \ell}\right)=\underbrace{\xi_{k \ell} \diamond \cdots \diamond \xi_{k \ell}}_{m \text { times }} .
$$

Then every multi-index $\alpha$ is uniquely characterized by the collection $\left\{\left(k_{1}, \ell_{1}\right), \ldots,\left(k_{n}, \ell_{n}\right)\right\}$, called the characteristic set of $\alpha$, such that $k_{1} \leq k_{2} \leq \cdots \leq k_{n}, \ell_{i} \leq \ell_{i+1}$ if $k_{i}=k_{i+1}$, 
and

$$
\xi_{\alpha}=\frac{\xi_{k_{1} \ell_{1}} \diamond \xi_{k_{2} \ell_{2}} \diamond \cdots \diamond \xi_{k_{n} \ell_{n}}}{\sqrt{\alpha !}} .
$$

Proposition 3.3. (a) The collection $\left\{\xi_{\alpha}, \alpha \in \mathcal{J}\right\}$ is an orthonormal basis in the space of square integrable random variables that are measurable with respect to the $\sigma$-algebra $\mathcal{F}^{W}$ generated by the Brownian motions $W_{\ell}, \ell \geq 1$, on $[0, T]$.

(b) Let $\eta$ be a square-integrable $\mathcal{F}^{W}$-measurable random element with values in $L_{2}((0, T))$. Define

$$
\eta_{\alpha}(t)=\mathbb{E}\left(\eta_{k}(t) \xi_{\alpha}\right)
$$

Then

$$
\mathfrak{X}_{\ell}(\eta)=\sum_{\alpha \in \mathcal{J}}\left(\sum_{k \geq 1} \sqrt{\alpha_{k \ell}} \int_{0}^{T} \eta_{\alpha-\epsilon_{k \ell}}(s)\left(\mathcal{K}_{\ell} h_{k}\right)(s) d s\right) \xi_{\alpha}
$$

as long as the series in $\alpha$ converges in the mean square (the inner sum always contains finitely many nonzero terms).

Proof. Part (a) is a classical result of Cameron and Martin [11. Part (b) follows from the definition of the Itô-Skorokhod integral in terms of the Wick product [29].

Let us now assume that equation (3.1) has a variational solution $u$ and

$$
u(t)=\sum_{\alpha \in \mathcal{J}} u_{\alpha}(t) \xi_{\alpha} .
$$

Substituting this representation into (3.1) and using (3.12), we conclude that each $u_{\alpha}$, which is nonrandom, satisfies

$$
\begin{aligned}
u_{\alpha}(t) & =u_{0, \alpha}+\int_{0}^{t}\left(\mathcal{A} u_{\alpha}(s)+F_{\alpha}(s)\right) d s \\
& +\sum_{k, \ell=1}^{\infty} \sqrt{\alpha_{k \ell}} \int_{0}^{t}\left(\mathcal{B}_{\ell} u_{\alpha-\epsilon_{k \ell}}(s)+G_{\ell, \alpha-\epsilon_{k \ell}}(s)\right)\left(\mathcal{K}_{\ell} h_{k}\right)(s) d s
\end{aligned}
$$

where $u_{0, \alpha}=\mathbb{E}\left(u_{0} \xi_{\alpha}\right), F_{\alpha}(t)=\mathbb{E}\left(F(t) \xi_{\alpha}\right), G_{\ell, \alpha}(t)=\mathbb{E}\left(G_{\ell}(t) \xi_{\alpha}\right)$. This observation motivates the following definition of the chaos solution.

Definition 3.4. (a) The collection of functions $\left\{u_{\alpha}, \alpha \in \mathcal{J}\right\}$ is called a chaos solution of equation (3.1) if every $u_{\alpha}$ is an element of $L_{2}((0, T) ; \mathbf{X})$ and the system of equalities (3.14) holds in $\mathbf{X}^{\prime}$ for all $t \in[0, T]$. The chaos solution is called square integrable if

$$
\sup _{0<t<T} \sum_{\alpha \in \mathcal{J}}\left\|u_{\alpha}(t)\right\|_{\mathbf{H}}^{2}<\infty .
$$

(b) The system of equalities (3.14) is called the S-system corresponding to equation (3.1).

REMARK 3.5. (a) If $\left\{u_{\alpha}, \alpha \in \mathcal{J}\right\}$ is a square integrable chaos solution, then, for each $t \in[0, T], u(t)=\sum_{\alpha \in \mathcal{J}} u_{\alpha}(t) \xi_{\alpha}$ is an element of $L_{2}(\Omega ; \mathbf{H})$, but still there is no guarantee that $u$ is a variational solution.

(b) Uniqueness of the chaos solution implies uniqueness of the variational solution. 
To establish existence and uniqueness of the chaos solution, we look at (3.1) as a system of equations. To solve this system, we make the following assumptions:

A1 The operator $\mathcal{A}$ is bounded linear from $\mathbf{X}$ to $\mathbf{X}^{\prime}$ and is strongly parabolic: there exist a positive number $\delta_{A}$ and a real number $C_{A}$ such that, for all $v \in \mathbf{X}$,

$$
\langle\mathcal{A} v, v\rangle+\delta_{A}\|v\|_{\mathbf{X}}^{2} \leq C_{A}\|v\|_{\mathbf{H}}^{2} .
$$

A2 Each $\mathfrak{X}_{\ell}, \ell \geq 1$, is a colored noise on $L_{2}((0, T))$ with a representation operator $\mathcal{K}_{\ell}$, and each $\mathcal{K}_{\ell}$ is a bounded linear operator on $L_{2}((0, T))$ with the operator $\operatorname{norm} \mathfrak{K}_{\ell}$ :

$$
\int_{0}^{T}\left|\left(\mathcal{K}_{\ell} f\right)(s)\right|^{2} d s \leq \mathfrak{K}_{\ell}^{2} \int_{0}^{T} f^{2}(s) d s, \quad f \in L_{2}((0, T)) .
$$

A3 The functions $u_{0}, F, G_{\ell}, \ell \geq 1$, are nonrandom and the $\mathfrak{X}_{\ell}, \ell \geq 1$, are jointly independent.

A4 Each $\mathcal{B}_{\ell}$ is a bounded linear operator on $\mathbf{H}$ with the operator norm $C_{\ell}$ :

$$
\left\|\mathcal{B}_{\ell} v\right\|_{\mathbf{H}} \leq C_{\ell}\|v\|_{\mathbf{H}}, v \in \mathbf{H} .
$$

A5 The following regularity conditions hold:

$$
\begin{gathered}
I_{0}=\left\|u_{0}\right\|_{\mathbf{H}}^{2}+\frac{2}{\delta_{A}} \int_{0}^{T}\|F(t)\|_{\mathbf{X}^{\prime}}^{2} d t+\sum_{\ell=1}^{\infty} \mathfrak{K}_{\ell}^{2} \int_{0}^{T}\left\|G_{\ell}(t)\right\|_{\mathbf{H}}^{2} d t<\infty, \\
C_{B}=\sum_{\ell=1}^{\infty} \mathfrak{K}_{\ell}^{2} C_{\ell}^{2}<\infty .
\end{gathered}
$$

Theorem 3.6. Under Assumptions A1-A5, equation (3.1) has a unique chaos solution. The solution is square integrable and satisfies

$$
\sup _{0<t<T} \mathbb{E}\|u(t)\|_{\mathbf{H}}^{2} \leq C_{o} e^{\left(C_{A}+C_{B}\right) T} I_{0},
$$

where $C_{A}>0$ is from (3.16) and $1 \leq C_{o} \leq 3$. In particular, $C_{o}=1$ if $F=G_{\ell}=0$.

Proof. Under Assumption A3, the S-system (3.14) corresponding to equation (3.1) becomes

$$
\begin{aligned}
& u_{(0)}(t)=u_{0}+\int_{0}^{t} \mathcal{A} u_{(0)}(s) d s+\int_{0}^{t} F(s) d s,|\alpha|=0 \\
& u_{\epsilon_{i j}}(t)=\int_{0}^{t} \mathcal{A} u_{\epsilon_{i j}}(s) d s+\int_{0}^{t}\left(\mathcal{B}_{j} u_{(0)}(s)+G_{j}(s)\right)\left(\mathcal{K}_{j} h_{i}\right)(s) d s,|\alpha|=1 \\
& u_{\alpha}(t)=\int_{0}^{t} \mathcal{A} u_{\alpha}(s) d s+\sum_{k, \ell=1}^{\infty} \sqrt{\alpha_{k \ell}} \int_{0}^{t} \mathcal{B}_{\ell} u_{\alpha-\epsilon_{k \ell}}(s)\left(\mathcal{K}_{\ell} h_{k}\right)(s) d s,|\alpha|>1 .
\end{aligned}
$$

Indeed, by Assumption A3, if $|\alpha|>0$, then $\mathbb{E}\left(u_{0} \xi_{\alpha}\right)=0, \mathbb{E}\left(F(t) \xi_{\alpha}\right)=0$, and $\mathbb{E}\left(G_{\ell} \xi_{\alpha}\right)=$ 0 .

The following proposition provides a key estimate for the solution of this system and is the main step in the proof of Theorem 3.6. 
Proposition 3.7. Under Assumptions A1-A6, for every $0 \leq t \leq T$ and $k \geq 1$,

$$
\begin{aligned}
\sum_{\substack{\alpha \in \mathcal{J} \\
|\alpha|=k}}\left\|u_{\alpha}(t)\right\|_{\mathbf{H}}^{2} & \leq C_{o} e^{C_{A} T}\left(\frac{\left(C_{B} T\right)^{k}}{k !}\left(\left\|u_{0}\right\|_{\mathbf{H}}^{2}+\frac{2}{\delta_{A}} \int_{0}^{T}\|F(s)\|_{\mathbf{X}^{\prime}}^{2} d s\right)\right. \\
& \left.+\frac{\left(C_{B} T\right)^{k-1}}{(k-1) !} \sum_{\ell=1}^{\infty} \mathfrak{K}_{\ell}^{2} \int_{0}^{T}\left\|G_{\ell}(s)\right\|_{\mathbf{H}}^{2} d s\right) .
\end{aligned}
$$

Proof. Given $v_{0} \in \mathbf{H}$ and $\psi \in L_{2}\left((0, T) ; \mathbf{X}^{\prime}\right)$, consider a deterministic evolution equation

$$
v(t)=v_{0}+\int_{0}^{t}(\mathcal{A} v(s)+\psi(s)) d s .
$$

By definition, $v \in L_{2}((0, T) ; \mathbf{X})$ is a solution of (3.24) if equality (3.24) holds in $\mathbf{X}^{\prime}$ for every $t \in[0, T]$.

It is known [37, Theorem 3.1.4] that, if the operator $\mathcal{A}$ is strongly parabolic, then:

- The operator $\mathcal{A}$ generates a semigroup $\Phi=\Phi_{t}, t \geq 0$, in the space $\mathbf{H}$.

- The semigroup $\Phi$ has the following properties:

$$
\begin{gathered}
\left\|\Phi_{t} v\right\|_{\mathbf{H}}^{2} \leq e^{C_{A} t}\|v\|_{\mathbf{H}}^{2}, v \in \mathbf{H} \\
\left\|\int_{0}^{t} \Phi_{t-s} f(s) d s\right\|_{\mathbf{H}}^{2} \leq \frac{2}{\delta_{A}} e^{C_{A} t} \int_{0}^{t}\|f(s)\|_{\mathbf{X}^{\prime}}^{2} d s, f \in L_{2}\left((0, T) ; \mathbf{X}^{\prime}\right) .
\end{gathered}
$$

- The solution of (3.24) is unique and can be written as

$$
v(t)=\Phi_{t} v_{0}+\int_{0}^{t} \Phi_{t-s} \psi(s) d s .
$$

We use these results to study the system of equations (3.22). It follows by induction on $|\alpha|$ that if $|\alpha|=k$ with the characteristic set $\left\{\left(i_{1}, \ell_{1}\right), \ldots,\left(i_{k}, \ell_{k}\right)\right\}$, and $\mathcal{P}_{k}$ is the set of all permutations of $\{1,2, \ldots, k\}$, then the solution of (3.22) is unique and is given by

$$
\begin{aligned}
u_{\alpha}(t)= & \frac{1}{\sqrt{\alpha !}} \sum_{\sigma \in \mathcal{P}_{k}} \int_{0}^{t} \int_{0}^{s_{k}} \ldots \int_{0}^{s_{2}} \Phi_{t-s_{k}} \mathcal{B}_{\ell_{\sigma(k)}} \ldots \Phi_{s_{2}-s_{1}}\left(\mathcal{B}_{\ell_{\sigma(1)}} u_{(0)}\left(s_{1}\right)\right. \\
& \left.+G_{\ell_{\sigma(1)}}\left(s_{1}\right)\right)\left(\mathcal{K}_{\ell_{\sigma(k)}} h_{i_{\sigma(k)}}\right)\left(s_{k}\right) \cdots\left(\mathcal{K}_{\ell_{\sigma(1)}} h_{i_{\sigma(1)}}\right)\left(s_{1}\right) d s_{1} \ldots d s_{k} .
\end{aligned}
$$

We then rewrite (3.28) as

$$
u_{\alpha}(t)=\int_{[0, T]^{k}} H\left(t, \ell^{(k)} ; s^{(k)}\right) \bar{h}_{\alpha}\left(s^{(k)}\right) d s_{1} \ldots d s_{k},
$$

where

$$
\begin{aligned}
H\left(t, \ell^{(k)} ; s^{(k)}\right) & =\frac{1}{\sqrt{k !}} \sum_{\sigma \in \mathcal{P}^{k}} \Phi_{t-s_{\sigma(n)}} \mathcal{B}_{\ell_{n}} \cdots \Phi_{s_{\sigma(2)}-s_{\sigma(1)}}\left(\mathcal{B}_{\ell_{1}} u_{(0)}\left(s_{\sigma(1)}\right)\right. \\
& \left.+G_{\ell_{1}}\left(s_{\sigma(1)}\right)\right) \chi_{s_{\sigma(2)}}\left(s_{\sigma(1)}\right) \cdots \chi_{t}\left(s_{\sigma(k)}\right)
\end{aligned}
$$

and

$$
\bar{h}_{\alpha}\left(s^{(k)}\right)=\frac{1}{\sqrt{\alpha ! k !}} \sum_{\sigma \in \mathcal{P}_{k}}\left(\mathcal{K}_{\ell_{1}} h_{i_{1}}\right)\left(s_{\sigma(1)}\right) \cdots\left(\mathcal{K}_{\ell_{k}} h_{i_{k}}\right)\left(s_{\sigma(k)}\right) .
$$


From (3.29) and the definition of the function $H$, we conclude that

$$
\begin{aligned}
& \sum_{\substack{\alpha \in \mathcal{J} \\
|\alpha|=k}}\left\|u_{\alpha}(t)\right\|_{\mathbf{H}}^{2} \leq \sum_{\ell_{1}, \ldots, \ell_{k}=1}^{\infty}\left(\prod_{j=1}^{k} \mathfrak{K}_{\ell_{j}}^{2}\right) \int_{0}^{t} \int_{0}^{s_{k}} \ldots \int_{0}^{s_{2}} \\
& \quad\left\|\Phi_{t-s_{k}} \mathcal{B}_{\ell_{k}} \ldots \Phi_{s_{2}-s_{1}}\left(\mathcal{B}_{\ell_{1}} u_{(0)}\left(s_{1}\right)+G_{\ell_{1}}\left(s_{1}\right)\right)\right\|_{\mathbf{H}}^{2} d s_{1} \ldots d s_{k} .
\end{aligned}
$$

By (3.22) and (3.27),

$$
u_{(0)}(t)=\Phi_{t} u_{0}+\int_{0}^{t} \Phi_{t-s} F(s) d s
$$

and then the properties (3.25) and (3.26) of the semigroup $\Phi$ imply that

$$
\left\|\mathcal{B}_{\ell} u_{(0)}(t)+G_{\ell}(t)\right\|_{\mathbf{H}}^{2} \leq C_{o}\left(C_{\ell}^{2} e^{C_{A} t}\left\|u_{0}\right\|_{\mathbf{H}}^{2}+\frac{2}{\delta_{A}} C_{\ell}^{2} e^{C_{A} t} \int_{0}^{t}\|F(s)\|_{\mathbf{X}^{\prime}}^{2} d s+\left\|G_{\ell}(t)\right\|_{\mathbf{H}}^{2}\right),
$$

where $C_{o}=3$, as the inequality $(a+b+c)^{2} \leq 3\left(a^{2}+b^{2}+c^{2}\right)$ suggests. On the other hand, $C_{o}=1$ if $F=G_{\ell}=0$. Applying (3.25) repeatedly, we find

$$
\begin{aligned}
& \left\|\Phi_{t-s_{k}} \mathcal{B}_{\ell_{k}} \cdots \Phi_{s_{2}-s_{1}}\left(\mathcal{B}_{\ell_{1}} u_{(0)}\left(s_{1}\right)+G_{\ell_{1}}\left(s_{1}\right)\right)\right\|_{\mathbf{H}}^{2} \\
& \leq C_{\ell_{k}}^{2} e^{C_{A}\left(t-s_{k}\right)}\left\|\Phi_{s_{k}-s_{k-1}} \mathcal{B}_{\ell_{k-1}} \cdots \Phi_{s_{2}-s_{1}}\left(\mathcal{B}_{\ell_{1}} u_{(0)}\left(s_{1}\right)+G_{\ell_{1}}\left(s_{1}\right)\right)\right\|_{\mathbf{H}}^{2} \leq \ldots \\
& \leq C_{o}\left(\prod_{j=2}^{k} C_{\ell_{j}}^{2}\right) e^{C_{A} t}\left(C_{\ell_{1}}^{2}\left\|u_{0}\right\|_{\mathbf{H}}^{2}+\frac{2}{\delta_{A}} C_{\ell_{1}}^{2} \int_{0}^{T}\|F(s)\|_{\mathbf{X}^{\prime}}^{2} d s+\left\|G_{\ell_{1}}\left(s_{1}\right)\right\|_{\mathbf{H}}^{2}\right) .
\end{aligned}
$$

Inequality (3.23) now follows from (3.32).

Proposition 3.7 is proved.

To complete the proof of Theorem 3.6 it remains to note that uniqueness of the chaos solution is equivalent to uniqueness of the solution of (3.22) and is guaranteed by the strong parabolicity of the operator $\mathcal{A}$. Then

$$
\sum_{\alpha \in \mathcal{J}}\left\|u_{\alpha}(t)\right\|_{\mathbf{H}}^{2}=\left\|u_{(0)}(t)\right\|_{\mathbf{H}}^{2}+\sum_{k \geq 1} \sum_{\substack{\alpha \in \mathcal{J} \\|\alpha|=k}}\left\|u_{\alpha}(t)\right\|_{\mathbf{H}}^{2},
$$

and (3.21) follows from (3.23).

As a first step toward studying the approximation of the chaos solution, we get

Corollary 3.8. Let $F=G_{\ell}=0$ and, for $N \geq 1$, define

$$
u_{N}(t)=\sum_{\substack{\alpha \in \mathcal{J} \\|\alpha| \leq N}} u_{\alpha}(t) \xi_{\alpha}
$$

Then

$$
\sup _{0<t<T} \mathbb{E}\left\|u(t)-u_{N}(t)\right\|_{\mathbf{H}}^{2} \leq \frac{\left(C_{B} T\right)^{N+1}}{(N+1) !} e^{\left(C_{A}+C_{B}\right) T}\left\|u_{0}\right\|_{\mathbf{H}}^{2} .
$$

Proof. We have

$$
\mathbb{E}\left\|u(t)-u_{N}(t)\right\|_{\mathbf{H}}^{2}=\sum_{\substack{\alpha \in \mathcal{J} \\|\alpha| \geq N+1}}\left\|u_{\alpha}(t)\right\|_{\mathbf{H}}^{2}
$$


because

$$
\mathbb{E}\left(\xi_{\alpha} \xi_{\beta}\right)= \begin{cases}1 & \text { if } \alpha=\beta, \\ 0 & \text { otherwise }\end{cases}
$$

Then (3.37) follows from (3.23).

REMARK 3.9. The conclusions of the theorem are valid even if the operators $\mathcal{A}, \mathcal{B}_{\ell}$ depend on time in a sufficiently regular way, as long as Assumptions A1 and A3 hold uniformly in $t \in[0, T]$.

There are at least two open problems related to the chaos solution of equation (3.1):

(1) To find out whether the solution belongs to

$$
L_{2}\left(\Omega ; L_{2}((0, T) ; X)\right) \cap L_{2}(\Omega ; C((0, T) ; H)) ;
$$

this is true when every $\mathfrak{X}_{\ell}$ is a white noise over $L_{2}((0, T))$ [28, Theorem 3.8].

(2) To establish existence of the solution when the operators $\mathcal{B}_{\ell}$ are unbounded on H.

\section{Approximation of the chaos solution.}

\subsection{One-step approximation.}

4.1.1. Motivation. If $\mathbf{V}$ is a Hilbert space, $f \in \mathbf{V}$, and $\left\{m_{k}, k \geq 1\right\}$ is an orthonormal basis in $\mathbf{V}$, then

$$
\sum_{k \geq 1}\left|\left(f, m_{k}\right)_{\mathbf{v}}\right|^{2}<\infty
$$

but nothing can be said about the rate of this convergence, that is, about the rate at which

$$
\sum_{k=n}^{\infty}\left|\left(f, m_{k}\right) \mathbf{v}\right|^{2}
$$

tends to zero as $n \rightarrow \infty$ : taking $\mathbf{V}=L_{2}((0, T))$ with a trigonometric basis, one can construct a function for which this convergence will be arbitrarily slow.

In the study of the chaos solution, we are facing a similar problem. In fact, the underlying Hilbert space is the space of square integrable random processes, the study of the rate of convergence for the "natural" approximations of the chaos solution is reduced to the analysis of certain Fourier series in $L_{2}((0, T))$.

As an illustration, consider the following equation:

$$
u(t, x)=u_{0}+\int_{0}^{t} u_{x x}(s, x) d s+\mathfrak{X}\left(\chi_{t} h(\cdot, x) u(\cdot, x)\right), t \geq 0, x \in \mathbb{R},
$$

where $\mathfrak{X}$ is a colored noise on $L_{2}((0, T))$ with representation $(\mathcal{K}, W)$.

If $u_{0} \in L_{2}(\mathbb{R})$ is nonrandom, and $h=h(t, x)$ is a bounded nonrandom function, then Theorem 3.6 implies that (4.1) has a unique square-integrable chaos solution $u(t, x)=$ $\sum_{\alpha \in \mathcal{I}} u_{\alpha}(t, x) \xi_{\alpha}$, where

$$
\begin{aligned}
\frac{\partial u_{(0)}}{\partial t} & =\frac{\partial^{2} u_{(0)}}{\partial x^{2}}, u_{(0)}(0, x)=u_{0}(x), \\
\frac{\partial u_{\alpha}}{\partial t} & =\frac{\partial^{2} u_{\alpha}}{\partial x^{2}}+\sum_{k=1}^{\infty} \sqrt{\alpha_{k}} h u_{\alpha-\epsilon_{k}} \mathcal{K} m_{k}, u_{\alpha}(0, x)=0,|\alpha|>0,
\end{aligned}
$$


and $\left\{m_{k}, k \geq 1\right\}$ is an orthonormal basis in $L_{2}((0, T))$; with only one colored noise driving the equation, every multi-index has the form $\alpha=\left\{\alpha_{1}, \alpha_{2}, \ldots\right\}$.

Denoting the heat semigroup by $\Phi_{t}$, we find

$$
u_{(0)}(t)=\Phi_{t} u_{0}(x)
$$

and

$$
u_{\epsilon_{k}}(t, x)=\int_{0}^{t} \Phi_{t-s} h \Phi_{s} u_{0}(s, x)\left(\mathcal{K} m_{k}\right)(s) d s .
$$

Let us define an approximation $u_{1}^{n}(t, x)$ of $u(t, x)$ by

$$
u_{1}^{n}(t, x)=u_{(0)}(t, x)+\sum_{k=1}^{n} u_{\epsilon_{k}}(t, x) \xi_{k} .
$$

What can we say about the quality of this approximation? For example, can we find a bound on $\sup _{0<t<T} \mathbb{E}\left\|u-u_{1}^{n}\right\|_{L_{2}(\mathbb{R})}^{2}(t)$ in terms of $n$ and $T$ ?

Since $u(t, x)=\sum_{\alpha \in \mathcal{I}} u_{\alpha}(t, x) \xi_{\alpha}$ and (3.39) holds, we have

$$
\mathbb{E}\left\|u-u_{1}^{n}\right\|_{L_{2}(\mathbb{R})}^{2}(t)=\sum_{\alpha \in \mathcal{I},|\alpha|>1}\left\|u_{\alpha}\right\|_{L_{2}(\mathbb{R})}^{2}(t)+\sum_{k=n+1}^{\infty}\left\|u_{\epsilon_{k}}\right\|_{L_{2}(\mathbb{R})}^{2}(t) .
$$

Using the properties of the heat semigroup on $\mathbb{R}$ and Corollary 3.8 , we find

$$
\sup _{0<t<T} \sum_{\alpha \in \mathcal{I},|\alpha|>1}\left\|u_{\alpha}\right\|_{L_{2}(\mathbb{R})}^{2}(t) \leq\left(C_{B} T\right)^{2} e^{C_{B} T},
$$

where $C_{B}=\|\mathcal{K}\| \sup _{t, x}|h(t, x)|^{2}$.

As a result, to find the quality of the approximation, we need to find the rate of convergence of the series $\sum_{k=1}^{\infty}\left\|u_{\epsilon_{k}}\right\|_{L_{2}(\mathbb{R})}^{2}(t)$. This rate of convergence is determined by the rate of decay, as $k \rightarrow \infty$, of $\left\|u_{\epsilon_{k}}\right\|_{L_{2}(\mathbb{R})}^{2}(t)$, and, as equality (4.4) suggests, one way to determine this rate is to integrate by parts. Accordingly, setting

$$
\widetilde{M}_{k}(t)=\int_{0}^{t}\left(\mathcal{K} m_{k}\right)(s) d s
$$

and using the properties of the heat semigroup, we get

$$
\begin{aligned}
u_{\epsilon_{k}}(t, x) & =\left.\Phi_{t-s}\left(h(s, \cdot) u_{(0)}(s, \cdot)\right)(x) \widetilde{M}_{k}(s)\right|_{s=0} ^{s=t} \\
& -\int_{0}^{t}\left(\Phi_{t-s} h\left(\Phi_{s} u_{0}\right)_{x x}-\left(\Phi_{t-s} h \Phi_{s} u_{0}\right)_{x x}\right) \widetilde{M}_{k}(s) d s .
\end{aligned}
$$

Note that (4.8) and the Cauchy-Schwarz inequality imply

$$
\left|\widetilde{M}_{k}(t)\right| \leq C \sqrt{t}
$$

and so $\widetilde{M}_{k}(0)=0$. Still, to advance our study of the rate of convergence any further, we need

(1) additional regularity of $u_{0}$ and $h$;

(2) rather detailed information about the functions $\widetilde{M}_{k}$. 
If we indeed assume all the necessary regularity of $u_{0}$ and $h$, then (4.9), together with the Cauchy-Schwarz inequality, implies

$$
\left\|u_{\epsilon_{k}}\right\|_{L_{2}(\mathbb{R})}^{2}(t) \leq C_{1}\left\|u_{0}\right\|_{L_{2}(\mathbb{R})}^{2}\left|\widetilde{M}_{k}(t)\right|^{2}+C_{2}\left\|\frac{\partial^{2} u_{0}}{\partial x^{2}}\right\|_{L_{2}(\mathbb{R})}^{2} t \int_{0}^{t}\left|\widetilde{M}_{k}(s)\right|^{2} d s .
$$

To continue, assume that

$$
m_{1}(s)=\frac{1}{\sqrt{T}} ; m_{k}(t)=\sqrt{\frac{2}{T}} \cos \left(\frac{\pi(k-1) t}{T}\right), k>1 ; 0 \leq t \leq T .
$$

With this choice of the basis, we use (4.8) to find that, for $k>1$ :

(1) If $\mathfrak{X}$ is white noise $(\mathcal{K}=I)$, then

$$
\widetilde{M_{k}}(t)=\frac{\sqrt{2 T}}{\pi(k-1)} \sin \left(\frac{\pi(k-1) t}{T}\right) .
$$

(2) If $\mathfrak{X}$ is the Ornstein-Uhlenbeck noise with parameter $b$, then

$$
\begin{aligned}
\widetilde{M}_{k}(t) & =\frac{\sqrt{2 T^{3}}}{b^{2} T^{2}+(k-1)^{2} \pi^{2}}\left(b \cos \left(\frac{\pi(k-1) t}{T}\right)-b e^{-b t}\right. \\
& \left.+\frac{(k-1) \pi}{T} \sin \left(\frac{\pi(k-1) t}{T}\right)\right) .
\end{aligned}
$$

(3) If $\mathfrak{X}$ the $H$-fractional white noise and $1 / 2<H<1$, then

$$
\left|\widetilde{M}_{k}(t)\right| \leq \frac{C(H) t^{2 H-1} T^{1-H}}{k^{\frac{3}{2}-H}}
$$

for some number $C(H)$ depending only on $H$.

Relation (4.11) suggests that the rate of convergence will be quite different for different $\mathfrak{X}$, and we are essentially forced to make the following assumptions about the functions $\widetilde{M}_{k}$ :

$$
\begin{gathered}
\sup _{0<t<T}\left|\widetilde{M}_{k}(t)\right|^{2} \leq \widetilde{C} \frac{T^{\delta}}{k^{\gamma}} \quad \text { for } \delta>0, \gamma>1, \widetilde{C}>0, \\
\left|\widetilde{M}_{k}(T)\right|^{2} \leq \widetilde{C} \frac{T^{\delta_{1}}}{k^{\gamma_{1}}} \quad \text { for } \delta_{1}>0, \gamma_{1}>1, \widetilde{C}>0 .
\end{gathered}
$$

In both (4.16) and (4.17), the number $\widetilde{C}$ should not depend on $T$ or $k$.

It is enough to have (4.16) and (4.17) for some orthonormal basis $\left\{m_{k}, k \geq 1\right\}$ in $L_{2}((0, T))$, but for now the cosine basis (4.12) is the only example when these assumptions can be verified. Below, we summarize the results for the white noise $W$, fractional white noise $W^{H}, 1 / 2<H<1$, and the Ornstein-Uhlenbeck noise $U_{b}, b>0$, when the cosine basis (4.12) is used.

REMARK 4.1. (a) In the case of $W$, with $m_{k}$ as in (4.12), we have $\widetilde{M_{k}}(T)=0$ for all $k \geq 2$, and then indeed any choice of $\delta_{1}, \gamma_{1}$ will work in (4.17).

(b) Inequality (4.11) shows that assumptions (4.16) and (4.17) are close to necessary for the analysis of convergence of the chaos expansion. 
TABLe 1

\begin{tabular}{ccccc}
\hline \hline & $\delta$ & $\gamma$ & $\delta_{1}$ & $\gamma_{1}$ \\
& & & & \\
\hline$W$ & 1 & 2 & Any & Any \\
& & & & \\
\hline$W^{H}$ & $2 H$ & $3-2 H$ & $2 H$ & $3-2 H$ \\
& & & & \\
\hline$U_{b}$ & 1 & 2 & 3 & 4 \\
\hline \hline
\end{tabular}

(c) As equality (4.9) suggests, no further integration by parts will, in general, improve the rate of convergence.

Under assumption (4.16), we conclude from (4.11) that, for $n>1$,

$$
\sup _{0<t<T} \sum_{k=n}^{\infty}\left\|u_{\epsilon_{k}}\right\|_{L_{2}(\mathbb{R})}^{2}(t) \leq \widetilde{C}_{1}\left\|u_{0}\right\|_{L_{2}(\mathbb{R})}^{2} \frac{T^{\delta}}{n^{\gamma-1}}+\widetilde{C}_{2}\left\|\frac{\partial^{2} u_{0}}{\partial x^{2}}\right\|_{L_{2}(\mathbb{R})}^{2} \frac{T^{\delta+2}}{n^{\gamma-1}} .
$$

With assumption (4.17), we also get

$$
\sum_{k=n}^{\infty}\left\|u_{\epsilon_{k}}\right\|_{L_{2}(\mathbb{R})}^{2}(T) \leq \widetilde{C}_{1}\left\|u_{0}\right\|_{L_{2}(\mathbb{R})}^{2} \frac{T^{\delta_{1}}}{n^{\gamma_{1}-1}}+\widetilde{C}_{2}\left\|\frac{\partial^{2} u_{0}}{\partial x^{2}}\right\|_{L_{2}(\mathbb{R})}^{2} \frac{T^{\delta+2}}{n^{\gamma-1}} .
$$

Inequality (4.18) establishes an approximation error bound uniformly over the time interval $(0, T)$, while (4.19) gives the bound only at the end point. If $T$ is small, and if we can take $\delta_{1}>\delta, \gamma_{1}>\gamma$, which is the case for $\mathfrak{X}=\mathfrak{B}$ and $\mathfrak{X}=U_{b}$, then (4.19) provides a better error bound than (4.18) and is more suitable for analyzing a step-by-step approximation.

By combining (4.7) with either (4.18) or (4.19), we will get the overall bound on the approximation error; for white, fractional, or Ornstein-Uhlenbeck noise, we also use Table 1. For example, when $\mathfrak{X}=W^{H}, 1 / 2<H<1$, we have $\|\mathcal{K}\|^{2} \leq C(H) T^{2 H-1}$ (see Example 2.5) and therefore, for $T \leq 1$,

$$
\sup _{0<t<T} \mathbb{E}\left\|u-u_{1}^{n}\right\|_{L_{2}(\mathbb{R})}^{2}(t) \leq C^{*}\left(T^{4 H}+\frac{T^{2 H}}{n^{2-2 H}}\right) .
$$

In the next section, we extend this result to more general equations and more general approximations.

4.1.2. Truncation of the S-system. Consider the following evolution equation:

$$
u(t)=u_{0}+\int_{0}^{t} \mathcal{A} u(s) d s+\sum_{\ell=1}^{\infty} \mathfrak{X}_{\ell}\left(\chi_{t} \mathcal{B}_{\ell} u\right),
$$


and assume that this equation has a unique square-integrable chaos solution $u$ in a normal triple $\left(\mathbf{X}, \mathbf{H}, \mathbf{X}^{\prime}\right)$ of Hilbert spaces. We also assume that $u$ has the chaos expansion

$$
u(t)=\sum_{\alpha \in \mathcal{J}} u_{\alpha}(t) \xi_{\alpha} .
$$

As before, we assume that $u_{0}$ is deterministic and every colored noise $\mathfrak{X}_{\ell}$ has a representation $\left(\mathcal{K}_{\ell}, W_{\ell}\right)$.

The first step is to find a general method of constructing an approximation of $u$ given the expansion (4.22). A natural approximation is

$$
\bar{u}(t)=\sum_{\alpha \in \overline{\mathcal{J}}} u_{\alpha}(t) \xi_{\alpha}
$$

where $\overline{\mathcal{J}}$ is a finite subset of $\mathcal{J}$. To control the size of this finite set, we use three characteristics of a multi-index:

$$
|\alpha|=\sum_{k, \ell} \alpha_{k \ell}, \varpi(\alpha)=\max \left\{k: \alpha_{k \ell}>0\right\}, d(\alpha)=\max \left\{\ell: \alpha_{k \ell}>0\right\} .
$$

For example, if

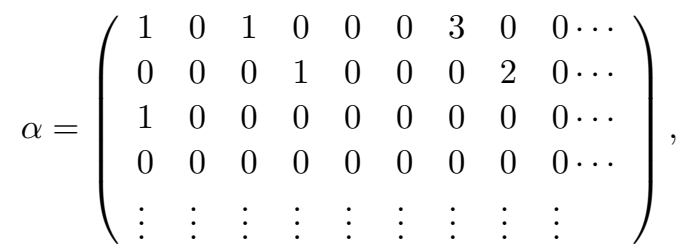

then $|\alpha|=1+1+3+1+2+1=9, \varpi(\alpha)=8, d(\alpha)=3$. We call $|\alpha|$ the length of the multi-index, $\varpi(\alpha)$ the order of the multi-index, and $d(\alpha)$ the dimension of the multi-index. Then the set

$$
\mathcal{J}_{N}^{n, r}=\{\alpha \in \mathcal{J}:|\alpha| \leq N, \varpi(\alpha) \leq n, d(\alpha) \leq r\}
$$

is finite, with no more than $(n r)^{N}$ elements. Note that the sets $\mathcal{J}_{N}=\{\alpha \in \mathcal{J}:|\alpha| \leq N\}$ are always infinite, and the set $\mathcal{J}_{N}^{n}=\{\alpha \in \mathcal{J}:|\alpha| \leq N, \varpi(\alpha) \leq n\}$ is infinite if and only if there are infinitely many noises in the equation. Accordingly, we define three approximations of $u$ :

$$
u_{N}(t)=\sum_{\alpha \in \mathcal{J}_{N}} u_{\alpha}(t) \xi_{\alpha}, u_{N}^{n}(t)=\sum_{\alpha \in \mathcal{J}_{N}^{n}} u_{\alpha}(t) \xi_{\alpha}, u_{N}^{n, r}(t)=\sum_{\alpha \in \mathcal{J}_{N}^{n, r}} u_{\alpha}(t) \xi_{\alpha} .
$$

Of the three, only $u_{N}^{n, r}(t)$ is computable, being a sum of finitely many terms. Consequently, our goal is to find a bound on $\sup _{0<t<T} \mathbb{E}\left\|u(t)-u_{N}^{n, r}(t)\right\|_{\mathbf{H}}$. Recall (see (3.14)) that the coefficients $u_{\alpha}$ satisfy the S-system

$$
\begin{aligned}
& u_{(0)}(t)=u_{0}+\int_{0}^{t} \mathcal{A} u_{(0)}(s) d s, \quad|\alpha|=0 \\
& u_{\alpha}(t)=\int_{0}^{t} \mathcal{A} u_{\alpha}(s) d s+\sum_{k, \ell=1}^{\infty} \sqrt{\alpha_{k \ell}} \int_{0}^{t} \mathcal{B}_{\ell} u_{\alpha-\epsilon_{k \ell}}(s)\left(\mathcal{K} m_{k}\right)(s) d s,|\alpha|>0 .
\end{aligned}
$$


By orthogonality of $\xi_{\alpha}$ for different $\alpha$, we have

$$
\begin{aligned}
\mathbb{E}\left\|u(t)-u_{N}^{n, r}(t)\right\|_{\mathbf{H}}^{2} & =\sum_{\alpha \notin \mathcal{J}_{N}^{n, r}} \mathbb{E}\left\|u_{\alpha}(t)\right\|_{\mathbf{H}}^{2}=\sum_{\alpha \in \mathcal{J} \backslash \mathcal{J}_{N}} \mathbb{E}\left\|u_{\alpha}(t)\right\|_{\mathbf{H}}^{2} \\
& +\sum_{\alpha \in \mathcal{J}_{N} \backslash \mathcal{J}_{N}^{n}} \mathbb{E}\left\|u_{\alpha}(t)\right\|_{\mathbf{H}}^{2}+\sum_{\alpha \in \mathcal{J}_{N}^{n} \backslash \mathcal{J}_{N}^{n, r}} \mathbb{E}\left\|u_{\alpha}(t)\right\|_{\mathbf{H}}^{2},
\end{aligned}
$$

where $\backslash$ denotes the difference of two sets. In other words, we have an analogue of the Pythagorean theorem:

$$
\begin{aligned}
\mathbb{E}\left\|u(t)-u_{N}^{n, r}(t)\right\|_{\mathbf{H}}^{2} & =\mathbb{E}\left\|u(t)-u_{N}(t)\right\|_{\mathbf{H}}^{2}+\mathbb{E}\left\|u_{N}(t)-u_{N}^{n}(t)\right\|_{\mathbf{H}}^{2} \\
& +\mathbb{E}\left\|u_{N}^{n}(t)-u_{N}^{n, r}(t)\right\|_{\mathbf{H}}^{2},
\end{aligned}
$$

and Corollary 3.8 provides an estimate for $\mathbb{E}\left\|u(t)-u_{N}(t)\right\|_{\mathbf{H}}^{2}$.

As we saw in the previous section, to estimate $\mathbb{E}\left\|u_{N}(t)-u_{N}^{n}(t)\right\|_{\mathbf{H}}^{2}$, we need to assume (4.16) and (4.17) together with additional regularity of the initial condition $u_{0}$ and the operators $\mathcal{A}, \mathcal{B}_{\ell}$. To formulate this regularity we need some additional constructions.

Let $\mathbf{H}^{r}, r \in \mathbb{R}$ be a scale of Hilbert spaces, or a Hilbert scale [25, Section VI.1.10] with the property that $\mathbf{H}^{0}=\mathbf{H}, \mathbf{H}^{1}=\mathbf{X}, \mathbf{H}^{-1}=\mathbf{X}^{\prime}$. A typical example of such a scale is the collection of the Sobolev spaces

$$
\mathbf{H}^{r}\left(\mathbb{R}^{d}\right)=\left\{f: \int_{\mathbb{R}^{d}}|\hat{f}(y)|^{2}\left(1+|y|^{2}\right)^{r} d y<\infty\right\},
$$

where $\hat{f}$ is the Fourier transform of $f$.

To generalize the computations that led to (4.11), we make the following assumptions:

$$
\begin{aligned}
& \mathbb{E}\left\|u_{0}\right\|_{\mathbf{H}^{2}}^{2}<\infty, \\
& \|\mathcal{A} v\|_{\mathbf{H}}^{2} \leq C_{02}\|v\|_{\mathbf{H}^{2}}^{2},\left\|\Phi_{t} v\right\|_{\mathbf{H}^{j}}^{2} \leq e^{C_{A} t}\|v\|_{\mathbf{H}^{j}}^{2}, j=0,2, \\
& \mathcal{B}_{\ell} \mathcal{B}_{n}=\mathcal{B}_{n} \mathcal{B}_{\ell} \text { for all } \ell, n, \\
& \left\|\mathcal{B}_{\ell} v\right\|_{\mathbf{H}^{2}}^{2} \leq C_{1, \ell}^{2}\|v\|_{\mathbf{H}^{2}}^{2}, v \in \mathbf{H}^{2}, \text { and } \sum_{\ell=1}^{\infty} C_{1, \ell}^{2} \mathfrak{K}_{\ell}^{2}=C_{1, B}<\infty,
\end{aligned}
$$

where $\Phi_{t}$ is the semigroup generated by $\mathcal{A}$.

Theorem 4.2. Assume that

- A1-A5 hold (see page 505);

- $F(t)=0$ and $G_{\ell}=0$;

- (4.16), (4.17) hold for all $\mathfrak{X}_{\ell}$ so that the numbers $\delta, \delta_{1}, \gamma, \gamma_{1}$, do not depend on $\ell$;

- (4.29) (4.32) hold.

Then

$$
\begin{aligned}
\sup _{0<t<T} \mathbb{E} \| u_{N}(t) & -u_{N}^{n}(t) \|_{\mathbf{H}}^{2} \\
& \leq C_{1, B} e^{\left(C_{A}+\bar{C}_{B}\right) T}\left(C_{B} \frac{T^{\delta}}{n^{\gamma-1}} \mathbb{E}\left\|u_{0}\right\|_{\mathbf{H}}^{2}+C_{02} C_{1, B} \frac{T^{\delta+2}}{n^{\gamma-1}} \mathbb{E}\left\|u_{0}\right\|_{\mathbf{H}^{2}}^{2}\right)
\end{aligned}
$$


and

$$
\begin{aligned}
\mathbb{E} \| u_{N}(T)- & u_{N}^{n}(T) \|_{\mathbf{H}}^{2} \\
& \leq C_{1, B} e^{\left(C_{A}+\bar{C}_{B}\right) T}\left(C_{B} \frac{T^{\delta_{1}}}{n^{\gamma_{1}-1}} \mathbb{E}\left\|u_{0}\right\|_{\mathbf{H}}^{2}+C_{02} C_{1, B} \frac{T^{\delta+2}}{n^{\gamma-1}} \mathbb{E}\left\|u_{0}\right\|_{\mathbf{H}^{2}}^{2}\right),
\end{aligned}
$$

where $\bar{C}_{B}=\max \left(C_{B}, C_{1, B}\right)$.

Proof. The argument is based on integration by parts in the representation of $u_{\alpha}(t)$ (see (3.29)); while the idea and the end result are essentially identical to (4.9), the computations are rather long. An interested reader can recover these computations following [27, where each $\mathfrak{X}$ is white noise.

REMARK 4.3. Similar to [27, a bound on $\mathbb{E}\left\|u_{N}(t)-u_{N}^{n}(t)\right\|_{\mathbf{H}}^{2}$ can be derived without condition (4.31), that is, if the operators $\mathcal{B}_{\ell}$ do not commute.

Finally, we derive a bound on $\mathbb{E}\left\|u_{N}^{n}(t)-u_{N}^{n, r}(t)\right\|_{\mathbf{H}}^{2}$.

Theorem 4.4. Assume that

- A1-A5 hold;

- $F(t)=0$ and $G_{\ell}=0$;

Define the sequence $\varepsilon=\varepsilon(r), r=1,2, \ldots$ by

$$
\sum_{\ell=r+1}^{\infty} \mathfrak{K}_{\ell}^{2} C_{\ell}^{2}=\varepsilon(r)
$$

Then

$$
\sup _{0<t<T} \mathbb{E}\left\|u_{N}^{n}(t)-u_{N}^{n, r}(t)\right\|_{\mathbf{H}}^{2} \leq \varepsilon(r) T e^{\left(C_{A}+C_{B}\right) T} \mathbb{E}\left\|u_{0}\right\|_{\mathbf{H}}^{2} .
$$

Proof. We have by (3.32),

$$
\begin{array}{r}
\mathbb{E}\left\|u_{N}^{n}(t)-u_{N}^{n, r}(t)\right\|_{\mathbf{H}}^{2} \leq \sum_{k=1}^{N} \sum_{j=1}^{k} \sum_{\ell_{j}=r+1}^{\infty} \sum_{\ell_{1}, \ldots, \ell_{k} \geq 1}^{(j)} \int_{0}^{t} \int_{0}^{s_{k}} \ldots \int_{0}^{s_{2}} \\
\left\|\Phi_{t-s_{k}} \mathcal{B}_{\ell_{k}} \cdots \Phi_{s_{2}-s_{1}} \mathcal{B}_{\ell_{1}} u_{(0)}\left(s_{1}\right)\right\|_{\mathbf{H}}^{2} d s_{1} \ldots d s_{k},
\end{array}
$$

where the summation $\sum_{\ell_{1}, \ldots, \ell_{k} \geq 1}^{(j)}$ omits the index $\ell_{j}$. Using the assumptions of the theorem, we conclude that

$$
\mathbb{E}\left\|u_{N}^{n}(t)-u_{N}^{n, r}(t)\right\|_{\mathbf{H}}^{2} \leq e^{C_{A} t}\left(\sum_{\ell=r+1}^{\infty} \mathfrak{K}_{\ell}^{2} C_{\ell}^{2}\right)\left(\sum_{k=1}^{\infty} k \frac{C_{B}^{k-1} t^{k}}{k !}\right) \mathbb{E}\left\|u_{0}\right\|_{\mathbf{H}}^{2},
$$

which implies (4.36).

REMARK 4.5. If there are finitely many $\mathfrak{X}_{\ell}$, and all of them are included in the approximation, then $\varepsilon(r)=0$ and $u_{N}^{n}(t)=u_{N}^{n, r}(t)$. 
Combining the results of Corollary 3.8 and Theorems 4.2 and 4.4 , we get the overall error bound:

$$
\begin{aligned}
\sup _{0<t<T} \mathbb{E} \| u(t) & -u_{N}^{n, r}(t) \|_{\mathbf{H}}^{2} \leq C(T)\left(\frac{\left(T C_{B}\right)^{N+1}}{(N+1) !} \mathbb{E}\left\|u_{0}\right\|_{\mathbf{H}}^{2}\right. \\
& \left.+\frac{T^{\delta}}{n^{\gamma-1}} \mathbb{E}\left\|u_{0}\right\|_{\mathbf{H}}^{2}+\frac{T^{\delta+2}}{n^{\gamma-1}} \mathbb{E}\left\|u_{0}\right\|_{\mathbf{H}^{2}}^{2}+T \varepsilon(r) \mathbb{E}\left\|u_{0}\right\|_{\mathbf{H}}^{2}\right),
\end{aligned}
$$

where $\lim _{T \rightarrow 0} C(T)>0$. A similar bound holds for $\mathbb{E}\left\|u(T)-u_{N}^{n, r}(T)\right\|_{\mathbf{H}}^{2}$ :

$$
\begin{aligned}
\mathbb{E} \| u(T) & -u_{N}^{n, r}(T) \|_{\mathbf{H}}^{2} \leq C(T)\left(\frac{\left(T C_{B}\right)^{N+1}}{(N+1) !} \mathbb{E}\left\|u_{0}\right\|_{\mathbf{H}}^{2}\right. \\
& \left.+\frac{T^{\delta_{1}}}{n^{\gamma_{1}-1}} \mathbb{E}\left\|u_{0}\right\|_{\mathbf{H}}^{2}+\frac{T^{\delta+2}}{n^{\gamma-1}} \mathbb{E}\left\|u_{0}\right\|_{\mathbf{H}^{2}}^{2}+T \varepsilon(r) \mathbb{E}\left\|u_{0}\right\|_{\mathbf{H}}^{2}\right),
\end{aligned}
$$

EXAmple 4.6. Consider the equation

$$
u(t)=u_{0}+\int_{0}^{t} u_{x x}(s) d s+\mathfrak{X}\left(\chi_{t} u\right), 0 \leq t \leq T, x \in \mathbb{R} .
$$

With only one noise driving the equation, we have $u_{N}^{n, r}=u_{N}^{n}$. Also, $\mathbf{H}^{r}=\mathbf{H}^{r}(\mathbb{R})$ is the Sobolev space (4.28) and $\mathbf{H}=L_{2}(\mathbb{R})$.

(a) If $\mathfrak{X}$ is an Ornstein-Uhlenbeck noise with parameter $b$, then $C_{B}=(1+\sqrt{b T})^{2}$, $\delta=1, \gamma=2$ (see Table 1). Inequality (4.37) becomes

$$
\begin{aligned}
\sup _{0<t<T} \mathbb{E} \| & u(t)-u_{N}^{n, r}(t) \|_{\mathbf{H}}^{2} \\
& \leq C_{b}(T)\left(\frac{(1+\sqrt{b T})^{2 N+2} T^{N}}{(N+1) !} \mathbb{E}\left\|u_{0}\right\|_{\mathbf{H}}^{2}+\frac{T}{n} \mathbb{E}\left\|u_{0}\right\|_{\mathbf{H}}^{2}+\frac{T^{3}}{n} \mathbb{E}\left\|u_{0}\right\|_{\mathbf{H}^{2}}^{2}\right),
\end{aligned}
$$

where $\lim \sup _{T \rightarrow 0} C_{b}(T)>0$.

(b) If $\mathfrak{X}$ is an $H$-fractional white noise with $H \in(1 / 2,1)$, then $C_{B}=C_{1}(H) T^{2 H-1}$, where

$$
C(H)=\frac{H(2 H-1) \Gamma\left(H-\frac{1}{2}\right)}{\Gamma\left(H+\frac{1}{2}\right)} ;
$$

see (2.3). Also, $\delta=2 H, \gamma=3-2 H$ (see Table 1). Inequality (4.37) becomes

$$
\begin{aligned}
& \sup _{0<t<T} \mathbb{E}\left\|u(t)-u_{N}^{n, r}(t)\right\|_{\mathbf{H}}^{2} \\
& \quad \leq C_{H}(T)\left(\frac{\left(C_{1}(H)\right)^{N+1} T^{2 H(N+1)}}{(N+1) !} \mathbb{E}\left\|u_{0}\right\|_{\mathbf{H}}^{2}+\frac{T^{2 H}}{n^{2-2 H}} \mathbb{E}\left\|u_{0}\right\|_{\mathbf{H}}^{2}+\frac{T^{2 H+2}}{n^{2-2 H}} \mathbb{E}\left\|u_{0}\right\|_{\mathbf{H}^{2}}^{2}\right),
\end{aligned}
$$

where $\lim \sup _{T \rightarrow 0} C_{H}(T)>0$.

4.2. Step-by-step approximation. 
4.2.1. Motivation. In the previous section, we constructed an approximate solution for equation (4.21) on the time interval $[0, T]$ and derived an error bound. The error bound suggests that the quality of the approximation improves for small values of $T$. To construct the approximation for large values of $T$, it is natural to use a step-by-step method.

The main idea of the step-by-step method is as follows. Let $\Psi_{t}$ be the solution operator for a homogeneous linear evolution equation; that is, given an initial condition $u_{0}, u(t)=$ $\Psi_{t} u_{0}$ is the solution of the equation at time $t$. If the equation is time-homogeneous (has no explicit dependence on time, such as (4.21) ) and the solution is unique, then the solution operator has the semigroup property:

$$
\Psi_{t} u_{0}=\Psi_{t-s} u(s), t>s>0 .
$$

If, for each $t>0, u(t)$ is an element of an infinite-dimensional Hilbert space $X$ with norm $\|\cdot\|$, then a one-step approximate solution $\bar{u}(t)$ can be constructed by

$$
\bar{u}(t)=\Pi^{N} \Psi_{t} u_{0},
$$

where $\Pi^{N}$ is an orthogonal projection on an $N$-dimensional subspace of $X$. Assume that the approximation is of order $p / 2$ in time for some $p>1$ :

$$
\|\bar{u}(t)-u(t)\|^{2}=\left\|\left(I-\Pi^{N}\right) \Psi_{t} u_{0}\right\|^{2} \leq C t^{p}\left\|u_{0}\right\|^{2},
$$

where $I$ denotes the identity operator. To construct a multi-step approximation on $[0, T]$, let $0=t_{0}<t_{1}<\ldots<t_{K}=T$ be a uniform partition of $[0, T]$ with step $\tau$. Then define $\bar{u}_{i}, i=0, \ldots, K$, recursively as follows:

$$
\bar{u}_{0}=u_{0}, \bar{u}_{i+1}=\Pi^{N} \Psi_{\tau} \bar{u}_{i} .
$$

For simplicity, we assume that the initial condition is not approximated and concentrate only on the effects of approximating the solution operator $\Psi_{t}$. Then, by linearity,

$$
u\left(t_{i}\right)-\bar{u}_{i}=\Psi_{\tau} u\left(t_{i-1}\right)-\Pi^{N} \Psi_{\tau} \bar{u}_{i-1}=\left(I-\Pi^{N}\right) \Psi_{\tau} u_{\tau_{i-1}}+\Pi^{N} \Psi_{\tau}\left(u_{\tau_{i-1}}-\bar{u}_{i-1}\right) .
$$

By orthogonality, we find

$$
\begin{aligned}
\left\|u\left(t_{i}\right)-\bar{u}_{i}\right\|^{2} & =\left\|\Psi_{\tau} u\left(t_{i-1}\right)-\Pi^{N} \Psi_{\tau} \bar{u}_{i-1}\right\|^{2} \\
& =\left\|\left(I-\Pi^{N}\right) \Psi_{\tau} u_{t_{i-1}}\right\|^{2}+\left\|\Pi^{N} \Psi_{\tau}\left(u_{t_{i-1}}-\bar{u}_{i-1}\right)\right\|^{2} .
\end{aligned}
$$

Let $\Delta_{i}=\left\|u\left(t_{i}\right)-\bar{u}_{i}\right\|^{2}$. Then (4.46) and (4.43) imply

$$
\Delta_{i} \leq C \tau^{p}\left\|u_{t_{i-1}}\right\|^{2}+\left\|\Psi_{\tau}\left(u_{\tau_{i-1}}-\bar{u}_{i-1}\right)\right\|^{2} .
$$

In many situations, the semigroup $\Psi_{t}$ satisfies

$$
\left\|\Psi_{t} f\right\| \leq e^{a t}\|f\|
$$

for some $a>0$. In this case, $\left\|u_{t_{i-1}}\right\|^{2} \leq e^{2 a T}\left\|u_{0}\right\|^{2}$ and (4.47) implies

$$
\Delta_{i} \leq C_{1} \tau^{p}\left\|u_{0}\right\|^{2}+e^{2 a \tau} \Delta_{i-1},
$$

or, after applying this inequality repeatedly,

$$
\Delta_{i} \leq C_{1} \tau^{p}\left\|u_{0}\right\|^{2} \sum_{j=0}^{i} e^{2 a \tau j} .
$$


Since

$$
\sum_{j=0}^{i} e^{2 a \tau j} \leq \sum_{j=0}^{K} e^{2 a \tau j}=\frac{e^{2 a(K+1) \tau}}{e^{2 a \tau}-1} \leq \frac{e^{4 a T}}{e^{2 a \tau}-1},
$$

and $e^{2 a \tau}-1 \geq 2 a \tau$, we conclude that

$$
\Delta_{i} \leq C_{2} \tau^{p-1}\left\|u_{0}\right\|^{2}
$$

that is,

$$
\max _{0 \leq i \leq K}\left\|u\left(t_{i}\right)-\bar{u}_{i}\right\|^{2} \leq C_{2} \tau^{p-1}\left\|u_{0}\right\|^{2}
$$

where $C_{2}$ depends only on $T$ and the semigroup $\Psi_{t}$. In other words, the step-by-step approximation has order $(p-1) / 2$ in time. The derivation of this result essentially relies on the following:

(1) an approximation based on an orthogonal projection;

(2) the property (4.48) of the solution operator.

4.2.2. The chaos solution. Let us consider equation (4.21). The approximation $u_{N}^{n, r}(t)$ of the solution is based on an orthogonal projection in the space of square-integrable processes and, by Theorem 3.6, the solution operator for the equation satisfies (4.48). We can therefore use (4.53) to derive an error bound for the step-by-step approximation of the solution of (4.21).

Let $0=t_{0}<t_{1}<\ldots<t_{K}=T$ be a uniform partition of the interval $[0, T]$ with step $\tau: t_{j}=j \tau, j=0, \ldots, K$. Let $\left\{m_{k}, k \geq 1\right\}$ be an orthonormal basis in $L_{2}((0, T))$ and $m_{k}^{j}(t)=m_{k}\left(t-t_{j}\right)\left(\chi_{t_{j+1}}(t)-\chi_{t_{j}}(t)\right)$. We define

$$
\xi_{k \ell}^{j}=\int_{t_{j-1}}^{t_{j}} m_{k}^{j}(t) d W_{\ell}(t)
$$

and then, for $\alpha \in \mathcal{J}$,

$$
\xi_{\alpha}^{j}=\prod_{k, \ell} \frac{H_{\alpha_{k \ell}}\left(\xi_{k \ell}^{j}\right)}{\sqrt{\alpha_{k \ell} !}} .
$$

Note that the random variables $\xi_{k \ell}^{i}$ and $\xi_{p q}^{j}$ are independent for different $i, j$.

If $u=u\left(t ; u_{0}\right)$ is the square-integrable chaos solution of the homogeneous equation (4.21) with initial condition $u_{0}$, then, by uniqueness and time homogeneity, we have

$$
u\left(t_{j} ; u_{0}\right)=u\left(\tau ; u\left(t_{j-1}, u_{0}\right)\right),
$$

which is a particular case of the general relation (4.42). Also, by Theorem 3.6.

$$
\mathbb{E}\left\|u\left(t ; u_{0}\right)\right\|_{\mathbf{H}}^{2} \leq e^{\left(C_{A}+C_{B}\right) t} \mathbb{E}\left\|u_{0}\right\|_{\mathbf{H}}^{2},
$$

which is a particular case of (4.48).

Next, consider the following modification of the S-system (4.25):

$$
\begin{aligned}
& u_{(0)}^{j}(t ; f)=f+\int_{t_{j-1}}^{t} \mathcal{A} u_{(0)}(s ; f) d s, \quad|\alpha|=0 ; \\
& u_{\alpha}^{j}(t ; f)=\int_{t_{j-1}}^{t} \mathcal{A} u_{\alpha}^{j}(s ; f) d s+\sum_{k, \ell=1}^{\infty} \sqrt{\alpha_{k \ell}} \int_{t_{j-1}}^{t} \mathcal{B}_{\ell} u_{\alpha-\epsilon_{k \ell}}^{j}(s ; f)\left(\mathcal{K} m_{k}^{j}\right)(s) d s
\end{aligned}
$$


for $|\alpha|>0$, where $t_{j-1} \leq t \leq t_{j}, \mathbb{E}\|f\|_{\mathbf{H}}^{2}<\infty$, and $f$ is random but independent of $\xi_{\alpha}^{j}, \alpha \in \mathcal{J}$.

Theorem 4.7. Assume A1-A5. Then, for $j=1, \ldots, K$,

$$
u\left(t_{j} ; u_{0}\right)=\sum_{\alpha \in \mathcal{J}} u_{\alpha}^{j}\left(t_{j} ; u\left(t_{j-1} ; u_{0}\right)\right) \xi_{\alpha}^{j}
$$

and

$$
\mathbb{E}\left\|u\left(t_{j} ; u_{0}\right)\right\|_{\mathbf{H}}^{2} \leq e^{\left(C_{A}+C_{B}\right) \tau} \mathbb{E}\left\|u\left(t_{j-1} ; u_{0}\right)\right\|_{\mathbf{H}}^{2} .
$$

Proof. Since $u\left(t_{j-1} ; u_{0}\right)$ is independent of $\left\{\xi_{\alpha}^{j}, \alpha \in \mathcal{J}\right\}$, Theorem 3.6 can be applied on each interval $\left[t_{j-1}, t_{j}\right], j=1, \ldots, K$, with $u_{0}$ replaced by $u\left(t_{j-1} ; u_{0}\right)$. Then (4.56) becomes (4.59) and (3.21) becomes (4.60).

Define the multi-step approximation of $u$ as follows:

$$
u_{N}^{n, r}\left(t_{j}\right)=\sum_{\alpha \in \mathcal{J}_{N}^{n, r}} u_{\alpha}^{j}\left(t_{j} ; u_{N}^{n, r}\left(t_{j-1}\right)\right) \xi_{\alpha}^{j}, j=1, \ldots, K,
$$

with $u_{N}^{n, r}\left(t_{0}\right)=u_{0}$. The following theorem provides an error bound for this approximation.

TheOREM 4.8. Under the assumptions of Theorems 4.2 and 4.4 .

$$
\begin{aligned}
& \max _{j=1, \ldots, K} \mathbb{E}\left\|u\left(t_{j} ; u_{0}\right)-u_{N}^{n, r}\left(t_{j}\right)\right\|_{\mathbf{H}}^{2} \\
\leq & C(T)\left(\frac{\left(\tau C_{B}\right)^{N}}{(N+1) !} \mathbb{E}\left\|u_{0}\right\|_{\mathbf{H}}^{2}+\frac{\tau^{\delta_{1}-1}}{n^{\gamma_{1}-1}} \mathbb{E}\left\|u_{0}\right\|_{\mathbf{H}}^{2}+\frac{\tau^{\delta}+1}{n^{\gamma-1}} \mathbb{E}\left\|u_{0}\right\|_{\mathbf{H}^{2}}^{2}+\varepsilon(r) \mathbb{E}\left\|u_{0}\right\|_{\mathbf{H}}^{2}\right) .
\end{aligned}
$$

Proof. Since $u_{N}^{n, r}(\tau)$ is an orthogonal projection of $u\left(\tau ; u_{0}\right)$ on the span of $\xi_{\alpha}, \alpha \in$ $\mathcal{J}_{N}^{n, r}$, and (4.57) holds, the result follows from (4.38) and (4.53).

EXAMPle 4.9. Consider the equation

$$
u(t)=u_{0}+\int_{0}^{t} u_{x x}(s) d s+\mathfrak{X}\left(\chi_{t} u\right), 0 \leq t \leq T, x \in \mathbb{R} .
$$

With only one noise driving the equation, we have $u_{N}^{n, r}=u_{N}^{n}$. Also, $\mathbf{H}^{r}=\mathbf{H}^{r}(\mathbb{R})$ is the Sobolev space (4.28) and $\mathbf{H}=L_{2}(\mathbb{R})$.

(a) If $\mathfrak{X}$ is an Ornstein-Uhlenbeck noise with parameter $b$, then $C_{B}=(1+\sqrt{b \tau})^{2}$, $\delta=1, \gamma=2, \delta_{1}=3, \gamma_{1}=4$ (see Table 1), so that (4.62) becomes

$$
\begin{array}{rl}
\max _{j=1, \ldots, K} & \mathbb{E}\left\|u\left(t_{j} ; u_{0}\right)-u_{N}^{n, r}\left(t_{j}\right)\right\|_{\mathbf{H}}^{2} \\
& \leq C_{b}(T)\left(\frac{(1+\sqrt{b \tau})^{2 N+2} \tau^{N}}{(N+1) !} \mathbb{E}\left\|u_{0}\right\|_{\mathbf{H}}^{2}+\frac{\tau^{2}}{n^{3}} \mathbb{E}\left\|u_{0}\right\|_{\mathbf{H}}^{2}+\frac{\tau^{2}}{n} \mathbb{E}\left\|u_{0}\right\|_{\mathbf{H}^{2}}^{2}\right) .
\end{array}
$$

(b) If $\mathfrak{X}$ is an $H$-fractional white noise with parameter $H \in(1 / 2,1)$, then $C_{B}=$ $C_{1}(H) \tau^{2 H-1}$ (see (4.40) $), \delta=\delta_{1}=2 H, \gamma=\gamma_{1}=3-2 H$ (see Table 11), so that (4.62) 
becomes

$$
\begin{aligned}
\max _{j=1, \ldots, K} \mathbb{E}\left\|u\left(t_{j} ; u_{0}\right)-u_{N}^{n, r}\left(t_{j}\right)\right\|_{\mathbf{H}}^{2} & \leq C_{H}(T)\left(\frac{\left(C_{1}(H)\right)^{N+1} \tau^{2 H(N+1)-1}}{(N+1) !} \mathbb{E}\left\|u_{0}\right\|_{\mathbf{H}}^{2}\right. \\
& \left.+\frac{\tau^{2 H-1}}{n^{2-2 H}} \mathbb{E}\left\|u_{0}\right\|_{\mathbf{H}}^{2}+\frac{\tau^{2 H+1}}{n^{2-2 H}} \mathbb{E}\left\|u_{0}\right\|_{\mathbf{H}^{2}}^{2}\right)
\end{aligned}
$$

Acknowledgment. SVL is grateful to Professor Wendell Fleming for very fruitful discussions, and to the Division of Applied Mathematics at Brown University for hospitality and stimulating atmosphere.

\section{REFERENCES}

[1] E. Alòs, O. Mazet, and D. Nualart, Stochastic Calculus With Respect to Fractional Brownian Motion with Hurst Parameter Less Than $\frac{1}{2}$, Stochastic Process. Appl. 86 (2000), no. 1, 121-139. MR:1741199 (2000m:60059)

[2] , Stochastic Calculus With Respect to Gaussian Processes, Ann. Probab. 29 (2001), no. 2, 766-801. MR.1849177 (2002g:60083)

[3] A. Amirdjanova, Nonlinear Filtering with Fractional Brownian Motion, Appl. Math. Optim. 46 (2002), no. 2-3, 81-88. MR1944752 (2003j:60086)

[4] A. Amirdjanova and S. Chivoret, New method for optimal nonlinear filtering of noisy observations by multiple stochastic fractional integral expansions, Comput. Math. Appl. 52 (2006), no. 1-2, 161-178. MR2262164

[5] R. J. Barton and H. V. Poor, Signal Detection in Fractional Gaussian Noise, IEEE Trans. Infom. Theory 34 (1988), no. 5, part 1, 943-959. MR982805 (90e:94007)

[6] J. F. Bennaton, Discrete Time Galerkin Approximation to the Nonlinear Filtering Solution, J. Math. Anal. Appl. 110 (1985), no. 2, 364-383. MR805259 (86k:93147)

[7] A. Bensoussan, R. Glowinski, and R. Rascanu, Approximations of the Zakai Equation by the Splitting-up Method, SIAM J. Control Optim. 28 (1990), no. 6, 1420-1431. MR1075210(91m:65243)

[8] T. Björk and H. Hult, A Note on Wick Products and the Fractional Black-Scholes Model, Finance Stoch. 9 (2005), no. 2, 197-209. MR2211124 (2006j:91142)

[9] A. Le Breton, Filtering and Parameter Estimation in a Simple Linear System Driven by Fractional Brownian Motion, Statist. Probab. Lett. 38 (1998), no. 3, 263-274. MR1629915 (99c:60088)

[10] A. Budhiraja and G. Kallianpur, Approximations to the Solution of the Zakai Equation Using Multiple Wiener and Stratonovich Integral Expansions, Stochastics Stochastics Rep. 56 (1996), no. 3-4, 271-315. MR.1396764 (97d:60077)

[11] R. H. Cameron and W. T. Martin, The Orthogonal Development of Nonlinear Functionals in Series of Fourier-Hermite Functionals, Ann. of Math. 48 (1947), no. 2, 385-392. MR0020230 (8:523a)

[12] W. Dai and C. C. Heyde, Itô's Formula with Respect to Fractional Brownian Motion and its Application, J. Appl. Math. Stochastic Anal. 9 (1996), no. 4, 439-448. MR1429266 (98f:60104)

[13] L. Decreusefond and A. S. Üstünel, Stochasic Analysis of the Fractional Brownian Motion, Potential Anal. 10 (1998), no. 2, 177-214. MR.1677455 (2000b:60133)

[14] T. E. Duncan, Y. Hu, and B. Pasik-Duncan, Stochastic Calculus for Fractional Brownian Motion I: Theory, SIAM J. Control Optim. 38 (2000), no. 2, 582-612. MR1741154 (2001g:60129)

[15] I. M. Gelfand and N. J. Vilenkin, Generalized Functions IV: Applications of Harmonic Analysis, Academic Press, 1964. MR0173945 (30:4152)

[16] A. Germani and M. Piccioni, Semi-discretisation of stochastic partial differential equations on $\mathbf{R}^{d}$ by a finite element technique, Stochastics 23 (1988), no. 2, 131-148. MR928351 (89f:60063)

[17] T. Hida, H-H. Kuo, J. Potthoff, and L. Streit, White Noise. An Infinite-Dimensional Calculus, Kluwer, 1993. MR:1244577 (95f:60046)

[18] H. Holden, B. Øksendal, J. Ubøe, and T. Zhang, Stochastic Partial Differential Equations: A Modeling, White Noise Functional Approach, Birkhäuser, 1996. MR/1408433 (98f:60124)

[19] Y. Hu and B. Øksendal, Fractional White Noise Calculus and Applications to Finance, Infin. Dimens. Anal. Quantum Probab. Relat. Top. 6 (2003), no. 1, 1-32. MR1976868 (2004c:60194) 
[20] K. Ito, Approximation of the Zakai Equation for Nonlinear Filtering, SIAM J. Control Optim. 34 (1996), no. 2, 620-634. MR1377715 (97f:93045)

[21] K. Ito and B. L. Rozovskii, Approximation of the Kushner equation for nonlinear filtering, SIAM J. Control Optim. 38 (2000), no. 3, 893-915. MR.1756900 (2001b:93074)

[22] M. L. Kleptsyna, A. Le Breton, and M.-C. Roubaud, General Approach to Filtering with Fractional Brownian Noises: Application to Linear Systems, Stochastics Stochastics Rep. 71 (2000), no. 1-2, 119-140. MR.1813509 (2001k:93131)

[23] M. L. Kleptsyna, P. E. Kloeden, and V. V. Anh, Existence and Uniqueness Theorems for Stochastic Differential Equations with Fractal Brownian Motion, Problems Inform. Transmission 34 (1998), no. 4, 332-341. MR1793494 (2001k:60079)

[24] P. E. Kloeden and E. Platen, Numerical Solution of Stochastic Differential Equations, Springer, 1992. MR 1214374 (94b:60069)

[25] S. G. Krein, Yu. I. Petunin, and E. M. Semeonov, Interpolation of Linear Operators, AMS, 1982. MR0649411 (84j:46103)

[26] S. J. Lin, Stochastic Analysis of Fractional Brownian Motions, Stochastics Stochastics Rep. 55 (1995), no. 1-2, 121-140. MR.1382288 (97j:60095)

[27] S. V. Lototsky, R. Mikulevicius, and B. L. Rozovskii, Nonlinear Filtering Revisited: A Spectral Approach, SIAM J. Contr. Optim. 35 (1997), no. 2, 435-461. MR1436632 (98a:60049)

[28] S. V. Lototsky and B. L. Rozovskii, Wiener Chaos Solutions of Linear Stochastic Evolution Equations, Ann. Probab. 34 (2006), no. 2, 638-662. MR2223954 (2007b:60154)

[29] S. V. Lototsky and K. Stemmann, From Random Processes to Generalized Fields: A Unified Approach to Stochastic Integration, Submitted to Stochastic Processes and Their Applications.

[30] P. Major, Multiple Wiener-Itô Integrals. With Applications to Limit Theorems, Lecture Notes in Mathematics, vol. 849, Springer, 1981. MR611334 (82i:60099)

[31] P. Malliavin, Stochastic analysis, Springer, 1997. MR.1450093 (99b:60073)

[32] G. Milstein, Numerical Integration of Stochastic Differential Equations, Kluwer, 1995. MR1335454 (96e:65003)

[33] D. Nualart, Stochastic Integration With Respect to Fractional Brownian Motion and Applications, Stochastic models (Mexico City, 2002), Contemp. Math., vol. 336, Amer. Math. Soc., Providence, RI, 2003, pp. 3-39. MR2037156 (2004m:60119)

[34] D. Nualart, The Malliavin calculus and related topics, second ed., Probability and its Applications (New York), Springer-Verlag, Berlin, 2006. MR2200233 (2006j:60004)

[35] B. Øksendal, Stochastic differential equations, sixth ed., Universitext, Springer-Verlag, Berlin, 2003. MR2001996 (2004e:60102)

[36] V. Pipiras and M. S. Taqqu, Integration Questions Related to Fractional Brownian Motion, Probab. Theory Related Fields 118 (2000), no. 2, 251-291. MR1790083 (2002c:60091)

[37] B. L. Rozovskii, Stochastic Evolution Systems, Kluwer, 1990. MR1135324 (92k:60136)

[38] M. Zähle, Integration With Respect to Fractal Functions and Stochastic Calculus I, Probab. Theory Related Fields 111 (1998), no. 3, 333-374. MR1640795 (99j:60073) 